Kragujevac Journal of Mathematics

Volume 46(1) (2022), Pages 105-113.

\title{
EXTREMAL GRAPHS FOR EXPONENTIAL VDB INDICES
}

\author{
ROBERTO CRUZ ${ }^{1}$ AND JUAN RADA ${ }^{1}$
}

\begin{abstract}
We find the extremal graphs for the exponential of well known vertexdegree-based topological indices over $\mathcal{G}_{n}$, the set of graphs with $n$ non-isolated vertices.
\end{abstract}

\section{INTRODUCTION}

A topological index is a number associated to a graph (for motivation and chemical applications see $[2,10,11,16,17])$. One important class of topological indices are the so-called vertex-degree-based (VDB for short) topological indices, which strongly depend on the degree of the vertices of the graph $[1,3,6,8,9,12]$.

More precisely, let $\mathcal{G}_{n}$ be the set of graphs with $n$ non-isolated vertices. Consider the function $m: \mathcal{G}_{n} \rightarrow \mathbb{R}^{\frac{(n-1) n}{2}}$ defined as $m(G)=\left(m_{i j}(G)\right)_{(i, j) \in K}$ for every $G \in \mathcal{G}_{n}$, where

$$
K=\{(i, j) \in \mathbb{N} \times \mathbb{N}: 1 \leq i \leq j \leq n-1\}
$$

and $m_{i j}(G)$ is the number of edges in $G$ joining vertices of degree $i$ and $j$. We order $K$ lexicographically so that $m(G)$ is a vector of $\mathbb{R}^{\frac{(n-1) n}{2}}$, for each $G \in \mathcal{G}_{n}$. A VDB topological index over $\mathcal{G}_{n}$ is a function $\varphi: \mathcal{G}_{n} \rightarrow \mathbb{R}$ induced by a vector $\varphi=\left(\varphi_{i j}\right)_{(i, j) \in K} \in \mathbb{R}^{\frac{(n-1) n}{2}}$, defined as

$$
\varphi(G)=m(G) \cdot \varphi
$$

the dot product of $m(G)$ and $\varphi$ as vectors in $\mathbb{R}^{\frac{(n-1) n}{2}}[13]$. In other words,

$$
\varphi(G)=\sum_{(i, j) \in K} m_{i j}(G) \varphi_{i j}
$$

Key words and phrases. Vertex-degree-based topological indices, exponential topological indices, extremal graphs.

2010 Mathematics Subject Classification. Primary:05C35. Secondary: 05C07.

DOI 10.46793/KgJMat2201.105C

Received: March 12, 2019.

Accepted: September 03, 2019. 
for every $G \in \mathcal{G}_{n}$.

The best known VDB topological indices are the following:

(a) the First Zagreb index [7], denoted by $\mathcal{F} Z$ and defined as $\varphi_{i j}=i+j$ for all $(i, j) \in K$

(b) the Second Zagreb index [7], denoted by $\mathcal{S} Z$ and defined as $\varphi_{i j}=i j$;

(c) the Randić index [15], denoted by $\chi$ and defined as $\varphi_{i j}=\frac{1}{\sqrt{i j}}$;

(d) the Harmonic index [20], denoted by $\mathcal{H}$ and defined as $\varphi_{i j}=\frac{2}{i+j}$;

(e) the Geometric-Arithmetic index [18], denoted by $\mathcal{G A}$ and defined as $\varphi_{i j}=\frac{2 \sqrt{i j}}{i+j}$;

(f) the Sum-Connectivity index [19], denoted by $\mathcal{S} \mathcal{C}$ and defined as $\varphi_{i j}=\frac{1}{\sqrt{i+j}}$;

(g) the Atom-Bond-Connectivity index [4], denoted by $\mathcal{A B C}$ and defined as $\varphi_{i j}=$ $\sqrt{\frac{i+j-2}{i j}}$

(h) the Augmented Zagreb index [5], denoted by $\mathcal{A} Z$ and defined as $\varphi_{i j}=\left(\frac{i j}{i+j-2}\right)^{3}$.

In a recent paper [14], the exponential of a VDB topological index $\varphi=\left(\varphi_{i j}\right)_{(i, j) \in K}$ was introduced as $\psi=e^{\varphi} \in \mathbb{R}^{\frac{(n-1) n}{2}}$, defined as

$$
\psi_{i j}=e^{\varphi_{i j}}
$$

for all $(i, j) \in K$. Among other things, it was shown in [14] that the exponential VDB topological indices have good discrimination properties. In this paper we determine the extremal graphs for the exponentials of all the best known VDB topological indices listed above, over the set $\mathcal{G}_{n}$.

Consider the VDB topological index $\varphi=\left(\varphi_{i j}\right)_{(i, j) \in K} \in \mathbb{R}^{\frac{(n-1) n}{2}}$. The vector $\varphi$ can be viewed as a function $\varphi: K \rightarrow \mathbb{R}$, where $\varphi(x, y)=\varphi_{x y}$ for all $(x, y) \in K$. We define the auxiliary function $f_{\varphi}: K \rightarrow \mathbb{R}$ defined as $f_{\varphi}(x, y)=\frac{x y \varphi_{x y}}{x+y}$. In order to find the maximal and minimal values of $\varphi$ over $\mathcal{G}_{n}$, it is sufficiently to find the maximal and minimal values of $f_{\varphi}$ over $K[12]$. Recall that

$$
K_{\min }\left(f_{\varphi}\right)=\left\{(r, s) \in K: f_{\varphi}(r, s)=\min _{(i, j) \in K} f_{\varphi}(i, j)\right\}
$$

and

$$
K_{\max }\left(f_{\varphi}\right)=\left\{(p, q) \in K: f_{\varphi}(p, q)=\max _{(i, j) \in K} f_{\varphi}(i, j)\right\} .
$$

We use notations $K_{\min }^{c}\left(f_{\varphi}\right)=K-K_{\min }\left(f_{\varphi}\right)$ and $K_{\max }^{c}\left(f_{\varphi}\right)=K-K_{\max }\left(f_{\varphi}\right)$. In order to compute $K_{\min }\left(f_{\varphi}\right)$ and $K_{\max }\left(f_{\varphi}\right)$, we will assume that $\varphi$ is a real continuous and differentiable function defined over the compact set

$$
\widehat{K}=\{(x, y) \in \mathbb{R} \times \mathbb{R}: 1 \leq x \leq y \leq n-1\} .
$$

Hence, $f_{\varphi}: \widehat{K} \rightarrow \mathbb{R}$ defined as $f_{\varphi}(x, y)=\frac{x y \varphi_{x y}}{x+y}$ for all $(x, y) \in \widehat{K}$, is also continuous and differentiable over $\widehat{K}$. 


\section{Extremal Values of Exponentials of VDB Topological Indices}

Based on [12, Theorem 2.3] and [12, Theorem 2.7] we will compute the maximal and minimal values of the exponentials of the VDB topological indices listed in the Introduction.

Theorem 2.1. Let $e^{\mathcal{F Z}}$ be the exponential of the first Zagreb index $\mathcal{F}$. Then:

1. $K_{n}$ is the unique maximal graph over $\mathcal{G}_{n}$ with respect to $e^{\mathfrak{F Z}}$, with value $\frac{n-1}{2} n e^{2(n-1)}$;

2. if $n$ is even (resp. odd), $\frac{n}{2} K_{2}$ (resp. $\frac{n-3}{2} K_{2} \cup P_{3}$ ) is the unique minimal graph over $\mathcal{G}_{n}$ with respect to $e^{\mathcal{F Z}}$, with value $\frac{n}{2} e^{2}$ (resp. $\left.\frac{n-3}{2} e^{2}+2 e^{3}\right)$.

Proof. The associated function for $e^{\mathfrak{F z}}$ over $\widehat{K}$ is

$$
f_{e^{\mathcal{F Z}}}(x, y)=\frac{x y e^{x+y}}{x+y} .
$$

Note that

$$
\frac{\partial}{\partial x}\left(\frac{x y e^{x+y}}{x+y}\right)=y \frac{e^{x+y}}{(x+y)^{2}}\left(x^{2}+y x+y\right)>0,
$$

for all $(x, y) \in \widehat{K}$.

1. By (2.1), the greatest value of $f_{e^{\mathcal{F}}}$ over $\widehat{K}$ is attained in the diagonal

$$
D=\{(x, y) \in \widehat{K}: y=x\} \text {. }
$$

Note that

$$
\left(f_{e^{\mathcal{F Z}}}(x, x)\right)^{\prime}=\left(\frac{1}{2} x e^{2 x}\right)^{\prime}=\frac{1}{2} e^{2 x}(2 x+1)>0,
$$

for all $x \in[1, n-1]$. It follows that

$$
K_{\max }\left(f_{e^{\mathfrak{F Z}}}\right)=\{(n-1, n-1)\} .
$$

Now we apply [12, Theorem 2.3] to obtain

$$
e^{\mathcal{F Z}}(G) \leq n f_{e^{\mathcal{F Z}}}(n-1, n-1)=\frac{1}{2}(n-1) n e^{2(n-1)}=e^{\mathcal{F Z}}\left(K_{n}\right) .
$$

Moreover, equality is obtained if and only if $m_{r s}(G)=0$ for all $(r, s) \neq(n-1, n-1)$. This is precisely $G=K_{n}$.

2. By (2.1), the minimal value of $f_{e^{\mathfrak{F}}}$ over $\widehat{K}$ is attained in the vertical line

$$
V=\{(x, y) \in \widehat{K}: x=1\} \text {. }
$$

Note that

$$
\left(f_{e^{\mathfrak{F Z}}}(1, y)\right)^{\prime}=\left(y \frac{e^{y+1}}{y+1}\right)^{\prime}=\frac{e^{y+1}}{(y+1)^{2}}\left(y^{2}+y+1\right)>0
$$


for all $y \in[1, n-1]$. Hence,

$$
K_{\min }\left(f_{e^{\mathfrak{F} z}}\right)=\{(1,1)\} .
$$

It follows from [12, Theorem 2.3] that if $n$ is even, then

$$
e^{\mathfrak{F Z}}(G) \geq n f_{e^{\mathfrak{F Z}}}(1,1)=\frac{n}{2} e^{2}=e^{\mathfrak{F Z}}\left(\frac{n}{2} K_{2}\right),
$$

for all $G \in \mathcal{G}_{n}$. Furthermore, equality is obtained if and only if $m_{r s}(G)=0$ for all $(r, s) \neq(1,1)$. This is precisely $G=\frac{n}{2} K_{2}$.

Finally, assume that $n$ is odd. From (2.1) and (2.2) we deduce that

$$
f_{e^{\mathcal{F Z}}}(1,2)<f_{e^{\mathcal{F Z}}}(x, y),
$$

for all $(x, y) \in K$ different from $(1,1)$ and $(1,2)$. Hence, by [12, Theorem 2.7],

$$
\begin{aligned}
e^{\mathfrak{F Z}}(G) & \geq f_{e^{\mathfrak{F Z}}}(1,1)(n-3)+3 f_{e^{\mathcal{F Z}}}(1,2) \\
& =\frac{n-3}{2} e^{2}+2 e^{3} \\
& =e^{\mathfrak{F Z}}\left(\frac{n-3}{2} K_{2} \cup P_{3}\right),
\end{aligned}
$$

for all $G \in \mathcal{G}_{n}$. Equality occurs if and only if $G=\frac{n-3}{2} K_{2} \cup P_{3}$.

An identical argument as in the proof of Theorem 2.1 works for the exponential of the Second Zagreb index $\mathcal{S} Z$ and the Atom-Bond-Connectivity index $\mathcal{A B C}$. We state them without proof.

Theorem 2.2. Let $e^{\mathcal{S}}$ be the exponential of the Second Zagreb index Sz. Then:

(a) $K_{n}$ is the unique maximal graph over $\mathcal{G}_{n}$ with respect to $e^{\text {sz }}$, with value

$$
\frac{1}{2}(n-1) n e^{(n-1)^{2}}
$$

(b) if $n$ is even (resp. odd), $\frac{n}{2} K_{2}$ (resp. $\left.\frac{n-3}{2} K_{2} \cup P_{3}\right)$ is the unique minimal graph over $\mathcal{G}_{n}$ with respect to $e^{\mathcal{\delta} z}$, with value $\frac{n}{2} e\left(\right.$ resp. $\left.\frac{n-3}{2} e+2 e^{2}\right)$.

Theorem 2.3. Let $e^{\mathcal{A B C}}$ be the exponential of the $\mathcal{A B C}$ index. Then:

(a) $K_{n}$ is the unique maximal graph over $\mathcal{G}_{n}$ with respect to $e^{\mathcal{A B C}}$, with value

$$
\frac{1}{2}(n-1) n e^{\frac{1}{n-1} \sqrt{2(n-2)}}
$$

(b) if $n$ is even (resp. odd), $\frac{n}{2} K_{2}$ (resp. $\left.\frac{n-3}{2} K_{2} \cup P_{3}\right)$ is the unique minimal graph over $\mathcal{G}_{n}$ with respect to $e^{\mathcal{A B C}}$, with value $\frac{n}{2}$ (resp. $\left.\frac{n-3}{2}+2 e^{\frac{1}{\sqrt{2}}}\right)$.

We next examine the exponential of the Harmonic index.

Theorem 2.4. Let $e^{\mathcal{H}}$ be the exponential of the Harmonic index $\mathcal{H}$. Then:

1. $K_{n}$ is the unique maximal graph over $\mathcal{G}_{n}$ with respect to $e^{\mathcal{H}}$, with value

$$
\frac{1}{2}(n-1) n e^{\frac{1}{n-1}}
$$


2. $S_{n}$ is the unique minimal graph over $\mathcal{G}_{n}$ with respect to $e^{\mathcal{H}}$, with value $(n-1) e^{\frac{2}{n}}$. Proof. The associated function for $e^{\mathcal{H}}$ is

$$
f_{e^{\mathcal{H}}}(x, y)=\frac{x y e^{\frac{2}{x+y}}}{x+y} .
$$

Now

$$
\frac{\partial}{\partial x}\left(\frac{x y e^{\frac{2}{x+y}}}{x+y}\right)=y e^{\frac{2}{x+y}} \frac{-2 x+x y+y^{2}}{(x+y)^{3}}>0,
$$

for all $(x, y) \in \widehat{K}-\{(1,1)\}$ (in $(1,1)$ equals to zero).

1. By (2.3), the maximal value of $f_{e^{\mathscr{H}}}$ over $\widehat{K}$ is attained in the diagonal

$$
D=\{(x, y) \in \widehat{K}: y=x\}
$$

Note that

$$
\left(f_{e^{\mathcal{H}}}(x, x)\right)^{\prime}=\left(\frac{1}{2} x e^{\frac{1}{x}}\right)^{\prime}=\frac{1}{2 x} e^{\frac{1}{x}}(x-1)>0,
$$

for all $x \in(1, n-1]$. Hence,

$$
K_{\max }\left(f_{e^{\mathcal{H}}}\right)=\{(n-1, n-1)\} .
$$

Now we apply [12, Theorem 2.3] to obtain

$$
e^{\mathcal{H}}(G) \leq n f_{e^{\mathcal{H}}}(n-1, n-1)=\frac{1}{2}(n-1) n e^{\frac{1}{n-1}}=e^{\mathcal{H}}\left(K_{n}\right) .
$$

Moreover, equality holds if and only if $m_{r s}(G)=0$ for all $(r, s) \neq(n-1, n-1)$, i.e., $G=K_{n}$.

2. By (2.3), the minimal value of $f_{e^{\mathscr{H}}}$ over $\widehat{K}$ is attained in the vertical line

$$
V=\{(x, y) \in \widehat{K}: x=1\} .
$$

Note that

$$
\left(f_{e^{\mathcal{H}}}(1, y)\right)^{\prime}=\left(y \frac{e^{\frac{2}{y+1}}}{y+1}\right)^{\prime}=-e^{\frac{2}{y+1}} \frac{y-1}{(y+1)^{3}}<0,
$$

for all $y \in(1, n-1]$. It follows that,

$$
K_{\min }\left(f_{e^{\mathcal{H}}}\right)=\{(1, n-1)\}
$$

Now, by [12, Theorem 2.3],

$$
e^{\mathscr{H}}(G) \geq n f_{e^{\mathcal{H}}}(1, n-1)=(n-1) e^{\frac{2}{n}}=e^{\mathcal{H}}\left(S_{n}\right),
$$

for all $G \in \mathcal{G}_{n}$. Equality holds if and only if $m_{r s}(G)=0$ for all $(r, s) \neq(1, n-1)$, i.e., $G=S_{n}$. 
The extremal values of the exponential of the Randić index $\chi$ and the AugmentedZagreb $\mathcal{A} Z$ can also be computed using an identical argument as in the proof of Theorem 2.4. We state them without proof.

Theorem 2.5. ([14, Theorem 4.3]). Let $e^{\chi}$ be the exponential of the Randic index $\chi$. Then:

(a) $K_{n}$ is the unique maximal graph over $\mathcal{G}_{n}$ with respect to $e^{\chi}$, with value

$$
\frac{1}{2}(n-1) n e^{\frac{1}{n-1}}
$$

(b) $S_{n}$ is the unique minimal graph over $\mathcal{G}_{n}$ with respect to $e^{\chi}$, with value

$$
(n-1) e^{\frac{1}{\sqrt{n-1}}}
$$

Theorem 2.6. Let $e^{\mathcal{A z}}$ be the exponential of the Augmented-Zagreb index AZ. Then:

(a) $K_{n}$ is the unique maximal graph over $\mathcal{G}_{n}$ with respect to $e^{\mathcal{A z}}$, with value

$$
\frac{1}{2}(n-1) n e^{\left(\frac{(n-1)^{2}}{2(n-2)}\right)^{3}}
$$

(b) $S_{n}$ is the unique minimal graph over $\mathcal{G}_{n}$ with respect to $e^{\mathcal{A z}}$, with value

$$
(n-1) e^{\left(\frac{n-1}{n-2}\right)^{3}} \text {. }
$$

Next we consider the exponential of the Geometric-Arithmetic index $\mathcal{G A}$.

Theorem 2.7. Let $e^{\mathcal{G A}}$ be the exponential of the Geometric-Arithmetic index $\mathcal{G A}$. Then

(a) $K_{n}$ is the unique maximal graph over $\mathcal{G}_{n}$ with respect to $e^{\mathcal{G A}}$, with value $\frac{1}{2}(n-1) n e ;$

(b) If $n \leq 34$ is even (resp. odd), $\frac{n}{2} K_{2}$ (resp. $\frac{n-3}{2} K_{2} \cup P_{3}$ ) is the unique minimal graph over $\mathcal{G}_{n}$ with respect to $e^{\mathcal{G A}}$ with value $\frac{n}{2} e\left(\right.$ resp. $\left.\frac{n-3}{2} e+2 e^{\frac{2 \sqrt{2}}{3}}\right)$;

(c) If $n \geq 35$, then $S_{n}$ is the unique minimal graph over $\mathcal{G}_{n}$ with respect to $e^{\mathcal{A A}}$, with value $(n-1) e^{\frac{2}{n} \sqrt{n-1}}$.

Proof. The associated function for $e^{\mathcal{G A}}$ is

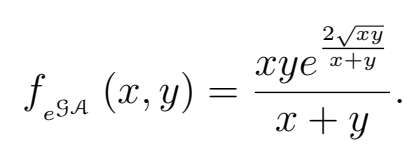

Note that

$$
\frac{\partial}{\partial x}\left(\frac{x y e^{\frac{2 \sqrt{x y}}{x+y}}}{x+y}\right)=y^{2} e^{2 \frac{\sqrt{x y}}{x+y}} \frac{\sqrt{x y}(x+y+\sqrt{x y})-x^{2}}{\sqrt{x y}(x+y)^{3}}>0,
$$

for all $(x, y) \in \widehat{K}$.

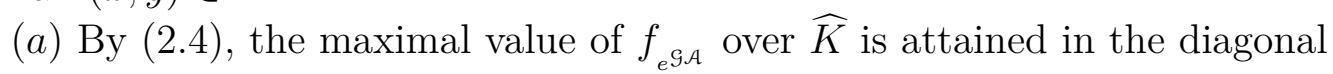

$$
D=\{(x, y) \in \widehat{K}: y=x\} \text {. }
$$


Note that

$$
\left(f_{e \mathcal{A}}(x, x)\right)^{\prime}=\left(\frac{1}{2} e x\right)^{\prime}=\frac{1}{2} e>0,
$$

for all $x \in[1, n-1]$. Hence,

$$
K_{\max }\left(f_{e^{\mathcal{G}}}\right)=\{(n-1, n-1)\} .
$$

It follows from [12, Theorem 2.3] that

$$
e^{\mathcal{G A}}(G) \leq n f_{e^{\mathcal{S A}}}(n-1, n-1)=\frac{1}{2}(n-1) n e,
$$

for all $G \in \mathcal{G}_{n}$. Equality holds if and only if $m_{r s}(G)=0$ for all $(r, s) \neq(n-1, n-1)$, i.e., $G=K_{n}$.

(b) By (2.4), the minimal value of $f_{e \mathcal{A}}$ over $\widehat{K}$ is attained in the vertical line

$$
V=\{(x, y) \in \widehat{K}: x=1\} \text {. }
$$

The graph of the one variable function $f_{e_{\mathcal{S A}}}(1, y)=\frac{y}{y+1} e^{\frac{2 \sqrt{y}}{y+1}}$ is shown in Figure 1 .

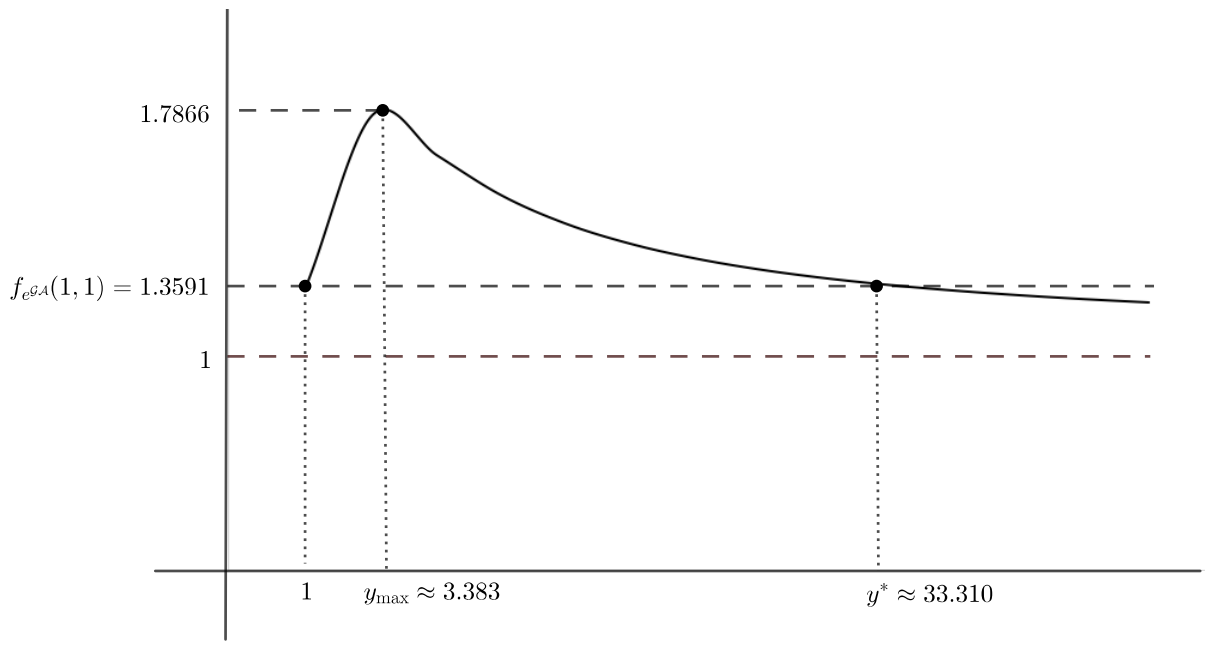

FiguRE 1. Graph of $f_{e^{\mathcal{A}}}(1, y)$.

The function $f_{e \mathcal{A}}(1, y)$ attains its maximal value at $y_{\max } \approx 3.383$, is strictly decreasing for $y>y_{\max }, f_{e \mathcal{G A}_{\mathcal{A}}}\left(1, y^{*}\right)=f_{e^{\mathcal{S}_{\mathcal{A}}}}(1,1)=1.3591$ for $y^{*} \approx 33.310$ and

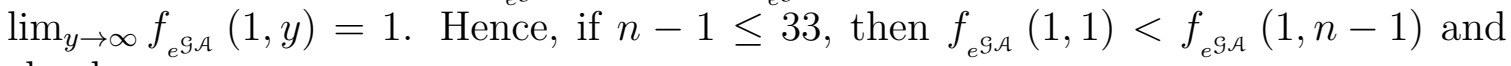
clearly

$$
K_{\min }\left(f_{e^{\mathcal{A}}}\right)=\{(1,1)\}
$$


A similar argument as in the proof of Theorem 2.1 shows that if $n \leq 34$ is even (resp. odd), then the minimal value of $e^{\mathcal{G A}}$ over $\mathcal{G}_{n}$ is attained uniquely in $\frac{n}{2} K_{2}$ (resp. $\left.\frac{n-3}{2} K_{2} \cup P_{3}\right)$ with value $\frac{n}{2} e$ (resp. $\frac{n-3}{2} e+2 e^{\frac{2 \sqrt{2}}{3}}$ ). On the other hand, if $n-1 \geq 34$, then

$$
K_{\min }\left(f_{e \mathcal{S A}}\right)=\{(1, n-1)\} .
$$

Now, by [12, Theorem 2.3],

$$
e^{\mathcal{G A}}(G) \geq n f_{e} \mathcal{G \mathcal { A }}(1, n-1)=(n-1) e^{\frac{2}{n} \sqrt{n-1}}=e^{\mathcal{H}}\left(S_{n}\right),
$$

for all $G \in \mathcal{G}_{n}$. Equality holds if and only if $m_{r s}(G)=0$ for all $(r, s) \neq(1, n-1)$, i.e., $G=S_{n}$.

A very similar argument to the one used in the proof of Theorem 2.7, gives the extremal values for the exponential of the Sum-Connectivity index SC. In the case of the minimal value of $e^{\mathcal{S E}}$ over $\widehat{K}$, the one variable function $f_{e^{s e}}(1, y)=\frac{y}{y+1} e^{\frac{1}{\sqrt{y+1}}}$, behaves similarly to the function $f_{e^{\mathcal{A}}}(1, y)$ for $y \geq 1$. It attains its maximal value at $y_{\max } \approx 4.8284$, is strictly decreasing for $y>y_{\max }, f_{e^{8 \mathcal{C}}}\left(1, y^{*}\right)=f_{e^{\text {se }}}(1,1)=1.0141$ for $y^{*} \approx 4986,3$ and $\lim _{y \rightarrow \infty} f_{e}$ se $(1, y)=1$. Hence, if $n-1 \leq 4986$, then $f_{e^{\text {se }}}(1,1)<$ $f_{\text {ese }}(1, n-1)$ and if $n-1 \geq 4987$, then $f_{e s e}(1, n-1)<f_{e s e}(1,1)$. We state it without proof.

Theorem 2.8. Let $e^{\text {se }}$ be the exponential of the Sum-Connectivity index SC. Then:

(a) $K_{n}$ is the unique maximal graph over $\mathcal{G}_{n}$ with respect to $e^{\text {se }}$, with value

$$
\frac{1}{2}(n-1) n e^{\frac{1}{\sqrt{2(n-1)}}}
$$

(b) if $n \leq 4987$ is even (resp. odd), $\frac{n}{2} K_{2}$ (resp. $\frac{n-3}{2} K_{2} \cup P_{3}$ ) is the unique minimal graph over $\mathcal{G}_{n}$ with respect to $e^{\text {se }}$ with value $\frac{n}{2} e^{\frac{1}{\sqrt{2}}}$ (resp. $\left.\frac{n-3}{2} e^{\frac{1}{\sqrt{2}}}+2 e^{\frac{1}{\sqrt{3}}}\right)$;

(c) if $n \geq 4988$, then $S_{n}$ is the unique minimal graph over $\mathcal{G}_{n}$ with respect to $e^{\text {se }}$, with value $(n-1) e^{\frac{1}{\sqrt{n}}}$.

\section{REFERENCES}

[1] R. Cruz, T. Pérez and J. Rada, Extremal values of vertex-degree-based topological indices over graphs, J. Appl. Math. Comput. 48 (2015), 395-406.

[2] J. Devillers and A. T. Balaban, Topological Indices and Related Descriptors, in: QSAR and QSPR, Gordon \& Breach, Amsterdam, 1999.

[3] T. Došlić, B. Furtula, A. Graovac, I. Gutman, S. Moradi and Z. Yarahmadi, On vertex-degreebased molecular structure descriptors, MATCH Commun. Math. Comput. Chem. 66 (2011), 613-626.

[4] E. Estrada, L. Torres, L. Rodríguez and I. Gutman, An atom-bond connectivity index: Modelling the enthalpy of formation of alkanes, Indian Journal of Chemistry 37A (1998), 849-855.

[5] B. Furtula, A. Graovac and D. Vukičević, Augmented Zagreb index, J. Math. Chem. 48 (2010), 370-380.

[6] B. Furtula, I. Gutman and M. Dehmer, On structure-sensitivity of degree-based topological indices, Appl. Math. Comput. 219 (2013), 8973-8978. 
[7] I. Gutman and N. Trinajstić, Graph theory and molecular orbitals. Total $\pi$-electron energy of alternant hydrocarbons, Chemical Physics Letters 17 (1972), 535-538.

[8] I. Gutman and J. Tošović, Testing the quality of molecular structure descriptors. Vertex-degreebased topological indices, Journal of the Serbian Chemical Society 78 (2013), 805-810.

[9] I. Gutman, Degree-based topological indices, Croatica Chemica Acta 86 (2013), 351-361.

[10] L. Kier and L. Hall, Molecular Connectivity in Chemistry and Drug Research, Academic Press, New York, 1976.

[11] L. Kier and L. Hall, Molecular Connectivity in Structure-Activity Analysis, Wiley, New York, 1986.

[12] J. Rada and R. Cruz, Vertex-degree-based topological indices over graphs, MATCH Commun. Math. Comput. Chem. 72 (2014), 603-616.

[13] J. Rada and S. Bermudo, Is every graph the extremal value of a vertex-degree-based topological index?, MATCH Commun. Math. Comput. Chem. 81 (2019), 315-323.

[14] J. Rada, Exponential vertex-degree-based topological indices and discrimination, MATCH Commun. Math. Comput. Chem. 82 (2019), 29-41.

[15] M. Randić, On characterization of molecular branching, Journal of the American Chemical Society 97 (1975), 6609-6615.

[16] R. Todeschini and V. Consonni, Handbook of Molecular Descriptors, Wiley-VCH, Weinheim, 2000.

[17] R. Todeschini and V. Consonni, Molecular Descriptors for Chemoinformatics, Wiley-VCH, Weinheim, 2009.

[18] D. Vukičević and B. Furtula, Topological index based on the ratios of geometrical and arithmetical means of end-vertex degrees of edges, J. Math. Chem. 46 (2009), 1369-1376.

[19] L. Zhong, The harmonic index for graphs, Appl. Math. Lett. 25 (2012), 561-566.

[20] B. Zhou and N. Trinajstić, On a novel connectivity index, J. Math. Chem. 46 (2009), 1252-1270.

\footnotetext{
${ }^{1}$ Instituto de Matematicas, UNIVERSIDAD DE ANTIOQUIA,

Medellín, Colombia

Email address: roberto.cruz@udea.edu.co

Email address: pablo.rada@udea.edu.co
} 